\title{
On the stability of nonlinear conservation laws in the Hausdorff metric ${ }^{1}$
}

\author{
Martin Campos-Pinto, Albert Cohen, Wolfgang Dahmen \\ and Ronald DeVore
}

\begin{abstract}
The mapping properties of the time evolution operator $E(t)$ of nonlinear hyperbolic scalar conservation laws is investigated. It is shown that this operator is Lipschitz in the Hausdorff metric in one space dimension whenever the flux is convex and one of the initial conditions satisfies a one-sided Lipschitz condition. The Hausdorff distance between the graphs of the solutions measures the closeness in $L^{\infty}$ in the regions where the solutions are smooth, as well as the closeness between the locations of shocks. A similar result on Hausdorff stability is proved with respect to a perturbation of the flux function. These results complement the well known $L^{1}$ contractivity of the solution operator. They are used in a subsequent paper to prove new smoothness results for solutions to such conservation laws. Negative results are proved in the case of non-convex and genuinly multidimensional fluxes.
\end{abstract}

Key Words: Conservation laws, Hausdorff metric, stability, one sided Lipschitz, perturbation of fluxes

AMS Subject Classification: 35L60, 35L65, 58K25

\section{Introduction}

A scalar hyperbolic conservation law on $\mathbb{R}^{\mathrm{d}}$ is the initial value problem

$$
\partial_{t} u+\operatorname{Div}_{x}(f(u))=0, \quad t>0, u(x, t=0)=u_{0}(x),
$$

\footnotetext{
${ }^{1}$ The research of R.D. was supported by the Office of Naval Research Contract Nr. N0014-91-J1343, the Army Research Office Contract Nr. DAAD 19-02-1-0028, the National Science Foundation Grant DMS0221642, the German Research Foundation (DFG) SFB 401, and by European Community's Human Potential Programme under contract HPRN-CT-202-00286, "Breaking Complexity"
} 
where $f: \mathbb{R} \rightarrow \mathbb{R}^{\mathrm{d}}$ is a $C^{1}$ function and $u: \mathbb{R}^{\mathrm{d}} \rightarrow \mathbb{R}$ is the solution. There are generally infinitely many weak solutions to (1) but appending an entropy condition, for example in the sense proposed by Kruzkov [5], extracts a unique solution $u(\cdot, t)$. We shall always be referring to this entropy solution when we speak of a solution to (1) and we shall denote by $E(t)$ the solution operator

$$
E(t) u_{0}:=u(\cdot, t)
$$

The operator $E(t)$ is a semigroup and has two fundamental properties:

1. Monotonicity : $u_{0} \leq v_{0}$ implies $E(t) u_{0} \leq E(t) v_{0}$.

2. $L^{1}$-contractivity : $\left\|E(t) u_{0}-E(t) v_{0}\right\|_{L^{1}} \leq\left\|u_{0}-v_{0}\right\|_{L^{1}}$.

For these and other properties of conservation laws we refer the reader to one of the many excellent texts [2], [4], [8].

On the other hand, the solution to (1) lacks classical smoothness and differentiability. For example, even if the initial condtion is smooth, the solution can develop discontinuities (called shocks) in finite time. It is interesting therefore to understand what can be said about the regularity of solutions to (1). The most famous result in this direction is the variation diminishing property:

$$
\left|E(t) u_{0}\right|_{\mathrm{BV}} \leq\left|u_{0}\right|_{\mathrm{BV}}
$$

which is easily derived from the $L^{1}$ contractivity. Here $B V=B V\left(\mathbb{R}^{\mathrm{d}}\right)$ is the space of functions of bounded variation. In the univariate case, one can also use the $L^{1}$ contractivity to derive higher order Besov regularity [3], and error estimates in the $L^{1}$ norm for numerical schemes [6].

Due to the appearance of discontinuities in the solution, no similar stability result is to be expected in the $L^{\infty}$ norm. In many instances however, one is interested in estimating the pointwise behavior of the solution. One possibility is to study the behavior of the solution and its approximations sufficiently away from the discontinuities, see in particular [10, 11, 12]. In this paper, we propose to measure the closeness between solutions by the Hausdorff distance between their graphs, i.e. given functions $u, v$ on $\mathbb{R}^{\mathrm{d}}$, we define

$$
d(u, v)=d_{H}\left(G_{u}, G_{v}\right)
$$


where $G_{v}$ denotes the graph of a function $v$ (discussed in more detail below) and

$$
d_{H}(A, B):=\max \left\{\sup _{a \in A} \inf _{b \in B}|a-b|, \sup _{b \in B} \inf _{a \in A}|a-b|\right\}
$$

is the Hausdorff distance between sets (with $|\cdot|$ denoting the Euclidean distance in $\left.\mathbb{R}^{\mathrm{d}+1}\right)$. The distance $d(u, v)$ appears as a natural substitute for the $L^{\infty}$ distance for discontinuous functions. For example for univariate functions, it measures, on the one hand, their closeness in $L^{\infty}$ in the regions where one of the functions (say $u$ ) is smooth enough since one easily checks that

$$
\|u-v\|_{L^{\infty}} \leq d(u, v)\left[\left\|u^{\prime}\right\|_{L^{\infty}}+1\right] .
$$

On the other hand, it measures how accurately a sharp transition in $u$ (as a function of $x$ ) is matched by a sharp transition in $v$.

Since, by necessity, we are dealing with discontinuous functions, some care must be taken in defining their graphs. Our positive results are proved for univariate functions and, in this case, we shall limit ourselves to functions $v$ which are continuous except for a countable number of isolated discontinuities. At each such discontinuity $x_{0}$, we assume that $v$ has both right and left hand limits. We shall call such functions $v$ admissible. The graph $G_{v}$ of such a function $v$ is the collection of points $\{(x, v(x): x \in \mathbb{R}\}$ together with the line segments $L_{x_{0}}:=\left\{\left(x_{0}, y\right): y_{\min } \leq y \leq y_{\max }\right\}$ where $y_{\min }$ is the smallest and $y_{\max }$ is the largest of the left and right limits of $v$ at $x_{0}$. For example, if $v$ is a monotone function, or more generally if $v$ is of bounded variation, then $v$ is admissible and $G_{v}$ is well defined in this way. We should remark that admissibility is a natural condition since even if an initial condition $u_{0}$ is not admissible the solution will be admissible at later time $t>0$ because of the Oleinik entropy condition.

Our main results concern the univariate problem (1). One result shows that if the flux $f$ is convex and one of the initial conditions $u_{0}, v_{0}$ is one-sided Lipschitz (which implies it is admissible) and the other is admissible, then the entropy solutions $u$ and $v$ at time $t$ satisfy

$$
d(u, v) \leq C(t) d\left(u_{0}, v_{0}\right),
$$

where $C(t)$ grows at most linearly with time. Similar to the results of [9] for the $L^{1}$ norm, we also prove that if $u$ and $v$ are solutions of (1) with strictly 
convex flux functions $f$ and $g$ respectively and identical admissible initial values, then

$$
d(u, v) \leq C(t)\left\|f^{\prime}-g^{\prime}\right\|_{L^{\infty}} .
$$

These results are limited to univariate conservation laws with convex fluxes. We shall exhibit counterexamples in the case of non-convex functions and in the multivariate case as well.

These stability properties can be used to prove new smoothness results for conservation laws. In [1], high order Besov smoothness for the graphs of solutions of (1) is established based on (7), following an approach similar to [3]. An interesting open question is to establish error estimates in the Hausdorff distance for numerical schemes such as Godunov, Lax-Friedrich, Minmod, ENO and WENO.

This paper is organized as follows. To orient the reader, we first treat the simple case of the $1 \mathrm{D}$ Burgers equation in $\S 2$. We then prove (7) for more general convex 1D fluxes in $\S 3$. We finally prove (8) in $\S 4$. In $\S 5$, we show that Hausdorff stability does not hold for non-convex fluxes. In $\S 6$, we give our negative results for for multidimensional fluxes which are not the product of a fixed vector with a one dimensional convex function.

\section{Hausdorff stability for the 1D Burgers equa- tion}

We begin in this section by treating the one dimensional Burgers equation which is (1) with flux $f(u)=u^{2} / 2$. We say that a function $v$ on $\mathbb{R}$ is onesided Lipschitz if there is an $e \in\{-1,1\}$ and some constant $M>0$ such that

$$
v(x+h e)-v(x) \leq M h, \quad h>0 .
$$

Any function satisfying such a one-sided Lipschitz condition with bounded support is necessarily of bounded variation and therefore admissible.

Theorem 1. If $u_{0}$ is any function with compact support which is one sided Lipschitz then for all admissible $v_{0}$ we have

$$
d(u, v) \leq C(t) d\left(u_{0}, v_{0}\right), \quad t>0
$$


with $C(t)=\left[(1+\tilde{M} t)^{2}+\tilde{M}^{2}\right]^{1 / 2}$ and $\tilde{M}:=2 M+1$.

Before proving this result, we shall show that the one sided Lipschitz condition (9) is unavoidable. To see this consider for some $\delta<<1$ the initial data

$$
u_{0}:=\chi_{[0, \delta]} \text { and } v_{0}:=\chi_{\left[0, \delta^{2}\right]} .
$$

We obviously have $d\left(u_{0}, v_{0}\right) \leq \delta$. At time $t=1$, the entropy solutions are given by

$$
u(x, 1)=x \chi_{\left[0,(2 \delta)^{1 / 2}\right]}(x) \text { and } v(x, 1)=x \chi_{[0, \sqrt{2} \delta]}(x),
$$

so that $d(u, v)$ is of the order $\delta^{1 / 2}>>\delta$. This proves that the stability property does not hold.

Proof of Theorem 1: Let $\delta:=d\left(u_{0}, v_{0}\right)$. We define

$$
u_{0}^{-}(x):=u_{0}(x+e \delta)-\tilde{M} \delta \text { and } u_{0}^{+}(x):=u_{0}(x-e \delta)+\tilde{M} \delta,
$$

and first prove that

$$
u_{0}^{-} \leq u_{0} \leq u_{0}^{+} \text {and } u_{0}^{-} \leq v_{0} \leq u_{0}^{+} .
$$

For example, from the one-sided Lipschitz condition (9), we have

$$
u_{0}(x)-u_{0}^{-}(x)=u_{0}(x)-u_{0}(x+e \delta)+\tilde{M} \delta \geq(\tilde{M}-M) \delta,
$$

so that the first lower inequality in (14) holds. The first upper inequality follows similarly. To see the second set of inequalities, given a point $P=$ $\left(x, v_{0}(x)\right)$ from the graph of $v_{0}$, there is a point $Q=\left(y, u_{0}(y)\right)$ at distance at most $\delta$ from $P$. We then have

$$
\begin{aligned}
v_{0}(x)-u_{0}^{-}(x) & \geq u_{0}(y)-u_{0}(x+e \delta)-\left|u_{0}(y)-v_{0}(x)\right|+\tilde{M} \delta \\
& \geq-M(2 \delta)-\delta+\tilde{M} \delta=0
\end{aligned}
$$

where we have used the one-sided Lipschitz condition (9) in bounding $u_{0}(y)-$ $u_{0}(x+e \delta)$. A similar proof establishes that $v_{0} \leq u_{0}^{+}$.

By monotonicity, we are ensured that

$$
u^{-} \leq u \leq u^{+} \text {and } u^{-} \leq v \leq u^{+},
$$


at any later time $t>0$, where $u^{-}$and $u^{+}$are the corresponding entropy solutions. Now $u^{+}$and $u^{-}$have the explicit form

$$
u^{-}(x, t)=u(x+e \delta+\tilde{M} \delta t, t)-\tilde{M} \delta
$$

and

$$
u^{+}(x, t)=u(x-e \delta-\tilde{M} \delta t, t)+\tilde{M} \delta .
$$

Indeed, this follows from the translation invariance of the equation combined with the following remark: if $u$ is an entropy solution of Burgers equation and $a \in \mathbb{R}$, then $\tilde{u}$ defined by

$$
\tilde{u}(x, t)=u(x-a t, t)+a,
$$

both satisfies Burgers equation in the weak sense and the Oleinik entropy condition, and is therefore the entropy solution for the initial condition $\tilde{u}_{0}=$ $u_{0}+a$. We thus have

$$
d(u, v) \leq \max \left\{d\left(u^{-}, u\right), d\left(u^{+}, u\right)\right\} \leq \delta\left[(1+\tilde{M} t)^{2}+\tilde{M}^{2}\right]^{1 / 2},
$$

which concludes the proof.

\section{Hausdorff stability for 1D conservation laws and convex fluxes}

We now consider the problem (1) with $d=1$ and $f(u)$ a $C^{2}$ function such that

$$
0 \leq f^{\prime \prime}(u) \leq B
$$

We shall use the following description of the solution $u$ to (1) given by Lax [7] based on results of Hopf. Given $x$ and $t>0$, we have

$$
u(x, t)=u_{0}(y(x, t))
$$

where $y=y(x, t)$ is a global minimizer of

$$
F_{\left(u_{0}, f\right)}(y, x, t):=F(y, x, t):=\int_{0}^{y} u_{0}(s) d s+t f^{*}\left(\frac{x-y}{t}\right),
$$


with $f^{*}$ the Legendre transform of $f$. We recall that since $f$ is convex and $C^{2}$, one has $\left(f^{*}\right)^{\prime}=\left(f^{\prime}\right)^{-1}$. The global minimum may not be uniquely attained. This corresponds to the existence of shocks: in this case, $u(\cdot, t)$ is multivalued at $x$ and its graph above $x$ coincides with the segment $\{x\} \times$ $\left[u_{0}\left(y_{\max }\right), u_{0}\left(y_{\min }\right)\right]$ where $y_{\min }$ and $y_{\max }$ are the minimal and maximal values of $y(x, t)$ achieving the infimum of $F(y, x, t)$. We begin with a lemma which examines the effect on $y(x, t)$ incurred from perturbations of the flux or the initial condition.

Lemma 1. Let $u_{0}$ be any admissible function and let $f, g$ be strictly convex fluxes satisfying

$$
0<A \leq f^{\prime \prime}, g^{\prime \prime} \leq B .
$$

Then for all $x \in \mathbb{R}$ and $t>0$ the following properties hold.

(i) If $y(x, t)$ is a minimizer for (24) when $F=F_{\left(u_{0}, f\right)}$ is defined with flux $f$ and initial condition $u_{0}$, then for all $\eta= \pm 1$ there exists a minimizer $\bar{y}(x, t)$ for (24) when $\bar{F}=F_{\left(u_{0}, g\right)}$ is defined with flux $g$ and initial condition $u_{0}$ such that

$$
\eta y(x, t) \leq \eta \bar{y}(x+\eta \varepsilon t B / A, t)
$$

where

$$
\varepsilon:=\left\|f^{\prime}-g^{\prime}\right\|_{L^{\infty}}
$$

(ii) If $y(x, t)$ is a minimizer for $(24)$ when $F=F_{\left(u_{0}, f\right)}$ is defined with flux $f$ and initial condition $u_{0}$, then for all $a \in \mathbb{R}$ there exists a minimizer $\tilde{y}(x, t)$ for $(24)$ when $\tilde{F}=F_{\left(u_{0}+a, f\right)}$ is defined with flux $f$ and initial condition $u_{0}+a$ such that

$$
(\operatorname{sign} a) y(x, t) \leq(\operatorname{sign} a) \tilde{y}(x+a B t, t) .
$$

Proof: We prove (i) in the case $\eta=1$; the case $\eta=-1$ is treated similarly. It suffices to prove that for any $z<y=y(x, t)$, we have

$$
K:=\bar{F}(z, x+\varepsilon t B / A, t)-\bar{F}(y, x+\varepsilon t B / A, t) \geq 0 .
$$

Since $f^{\prime \prime}, g^{\prime \prime} \geq A$ and $\left(f^{*}\right)^{\prime}=\left(f^{\prime}\right)^{-1},\left(g^{*}\right)^{\prime}=\left(g^{\prime}\right)^{-1}$, it follows that

$$
\left\|\left(g^{*}\right)^{\prime}-\left(f^{*}\right)^{\prime}\right\|_{L^{\infty}} \leq\left\|f^{\prime}-g^{\prime}\right\|_{L^{\infty}} / A=\varepsilon / A .
$$


Hence,

$$
\begin{aligned}
K \geq & \bar{F}(z, x+t \varepsilon B / A, t)-\bar{F}(y, x+t \varepsilon B / A, t) \\
& -F(z, x, t)+F(y, x, t) \\
= & \int_{z}^{y}\left[\left(g^{*}\right)^{\prime}\left(\frac{x+t \varepsilon B / A-s}{t}\right)-\left(f^{*}\right)^{\prime}\left(\frac{x-s}{t}\right)\right] d s \\
\geq & \int_{z}^{y}\left[\left(f^{*}\right)^{\prime}\left(\frac{x+t \varepsilon B / A-s}{t}\right)-\left(f^{*}\right)^{\prime}\left(\frac{x-s}{t}\right)\right] d s-\frac{1}{A} \varepsilon(y-z) \\
\geq & \inf \left(f^{*}\right)^{\prime \prime} \varepsilon \frac{B}{A}(y-z)-\frac{1}{A} \varepsilon(y-z) \geq 0,
\end{aligned}
$$

where we use the minimizing property of $y$ in the first inequality, (30) in the second one, the hypothesis $z \leq y$ in the third one, and the fact that $\inf \left(f^{*}\right)^{\prime \prime}=1 / \sup (f)^{\prime \prime}$ in the last one. This completes the proof of (i).

The proof of (ii) is similar. We assume that $a>0$ (the case $a<0$ is proved similarly). It suffices to show that for all $z<y=y(x, t)$, we have

$$
K:=\tilde{F}(z, x+a B t, t)-\tilde{F}(y, x+a B t, t) \geq 0 .
$$

As above we obtain

$$
\begin{aligned}
K \geq & \tilde{F}(z, x+a B t, t)-\tilde{F}(y, x+a B t, t)-F(z, x, t)+F(y, x, t) \\
= & \int_{z}^{y}\left(u_{0}-\tilde{u}_{0}\right)(s) d s+t\left(f^{*}\left(\frac{x-y}{t}\right)-f^{*}\left(\frac{x-z}{t}\right)\right. \\
& \left.\quad+f^{*}\left(\frac{x-y+a B t}{t}\right)-f^{*}\left(\frac{x-z+a B t}{t}\right)\right) \\
= & -a(y-z)+\int_{z}^{y}\left(\left(f^{*}\right)^{\prime}\left(\frac{x-s}{t}+a B\right)-\left(f^{*}\right)^{\prime}\left(\frac{x-s}{t}\right)\right) d s \\
\geq & -a(y-z)+\int_{z}^{y} a B \inf \left(f^{*}\right)^{\prime \prime} \\
\geq & -a(y-z)+a(y-z)=0,
\end{aligned}
$$

where we have again used $\inf \left(f^{*}\right)^{\prime \prime}=1 / \sup (f)^{\prime \prime}$.

We can now prove the following generalization of Theorem 1 .

Theorem 2. If $u_{0}$ satisfies the one-sided Lipschitz condition (9) then for any admissible $v_{0}$ we have

$$
d(u, v) \leq C(t) d\left(u_{0}, v_{0}\right),
$$


for all $t>0$, with $C(t)=\left[(1+\tilde{M} B t)^{2}+(\tilde{M} B / A)^{2}\right]^{1 / 2}$ and $\tilde{M}=\left(1+M^{2}\right)^{1 / 2}+$ $M$.

Proof: We define $u^{-}$and $u^{+}$in exactly the same way as in the proof of Theorem 1 with $\delta:=d\left(u_{0}, v_{0}\right)$. Then, as was shown in the proof in Theorem 1 , we have

$$
u^{-} \leq u, v \leq u^{+}
$$

We cannot conclude as easily as in Theorem 1, since we do not have an explicit formula for $u^{-}$and $u^{+}$in terms of $u$. However, we have $u^{ \pm}=$ $\tilde{u}^{ \pm}(\cdot \mp e \delta)$ where $\tilde{u}^{ \pm}$is the solution with initial data $\tilde{u}_{0}^{ \pm}:=u_{0} \pm a$. We shall first show that for all $t>0$ and $x \in \mathbb{R}$, the graph of $\tilde{u}^{+}(\cdot, t)$ passes through the rectangle $[x, x+a B t] \times[u(x, t), u(x, t)+a B / A]$ and the graph of $\tilde{u}^{-}(\cdot, t)$ passes through the rectangle $[x+a B t, x] \times[u(x, t)+a B / A, u(x, t)]$.

We consider the case of $\tilde{u}^{+}$, the case of $\tilde{u}^{-}$being treated in a similar way. Since by monotonicity, we have $\tilde{u}^{+}(x, t) \geq u(x, t)$, the rectangle $[x, x+a B t] \times$ $[u(x, t), u(x, t)+a B / A]$ is certainly intersected by the graph of $\tilde{u}^{+}$if this graph contains a point on the half vertical line $\{x+a B t\} \times]-\infty, u(x, t)+a B / A]$. In other words, we want to prove that

$$
\tilde{u}^{+}(x+a B t) \leq u(x, t)+a B / A,
$$

where $\tilde{u}^{+}(x+a B t)$ might be one of the multiple values of $\tilde{u}^{+}$at $x+a B t$ in the case of a discontinuity at this point.

We now invoke Lemma 1 part (ii) where $y=y(x, t)$ is the minimizer for $F$ in (24) with flux $f$ and initial condition $u_{0}$ that gives $u(x, t)=u_{0}(y)$ and $\tilde{y}$ is a minimizer for $\tilde{F}=F_{\left(u_{0}^{+}, f\right)}(\cdot, x+a B t, t)$ with flux $f$, initial condition $\tilde{u}_{0}^{+}=u_{0}+a$ that gives $\tilde{u}^{+}(x+a B t, t)=\tilde{u}_{0}^{+}(\tilde{y})$. The Lemma ensures that we can choose $\tilde{y}$ such that $y=y(x, t) \leq \tilde{y}(x+a B t)$. Since $y$ and $\tilde{y}$ satisfy $x-y=t f^{\prime}\left(u_{0}(y)\right)$ and $x+a B t-\tilde{y}=t f^{\prime}\left(\tilde{u}_{0}^{+}(\tilde{y})\right)$, we infer that

$$
f^{\prime}\left(\tilde{u}_{0}^{+}(\tilde{y})\right)-f^{\prime}\left(u_{0}(y)\right) \leq a B .
$$

which clearly implies (38) since $f^{\prime \prime} \geq A$.

By what we have shown, we conclude that for any point $(x, u(x, t))$ on the graph of $u(\cdot, t)$, there is a point $Q$ on the graph of $u^{+}$at a distance at most $C(t) d\left(u_{0}, v_{0}\right)$ from $P$. By reversing the roles of $u_{0}^{+}$and $u_{0}$ we conclude that for any $Q$ on the graph of $u^{+}$there is a point $P$ on the graph of $u$ at 
distance at most $C(t) d\left(u_{0}, v_{0}\right)$ from $Q$. Hence $d\left(u, u^{+}\right) \leq C(t) d\left(u_{0}, v_{0}\right)$. Similar reasoning gives $d\left(u, u^{-}\right) \leq C(t) d\left(u_{0}, v_{0}\right)$. In view of (33) we have proven the theorem.

Remark 1: In the statement of Theorem 2, we can take for $A$ and $B$ the minimum and maximum of $f^{\prime \prime}$ restricted to the interval $I:=\left[\min \left(u_{0}\right)-\right.$ $\left.\tilde{M} d\left(u_{0}, v_{0}\right), \max \left(u_{0}\right)+\tilde{M} d\left(u_{0}, v_{0}\right)\right]$.

Remark 2: Theorem 2 gives Theorem 1 with a slightly better constant in the case of Burgers equation for which $A=B=1$.

Theorem 2 can be generalized to convex fluxes.

Theorem 3. If the flux $f$ satisfies

$$
0 \leq f^{\prime \prime} \leq B
$$

and the initial condition $u_{0}$ satisfies the one-sided Lipschitz condition (9) with $e=1$, then

$$
d(u, v) \leq C(t) d\left(u_{0}, v_{0}\right),
$$

for all admissible $v_{0}$ and all $t>0$, with $C(t)=\left[(1+\tilde{M} B t)^{2}+(\tilde{M}(1+\right.$ $\left.t M B))^{2}\right]^{1 / 2}$ and $\tilde{M}=\left(1+M^{2}\right)^{1 / 2}+M$.

Proof: The proof follows the lines of Theorems 1 and 2. We define $u_{0}^{ \pm}=$ $u_{0}(\cdot \mp e \delta) \pm a$ and $\tilde{u}_{0}^{ \pm}:=u_{0} \pm a$ with again $a:=\tilde{M} \delta$ and $\delta:=d\left(u_{0}, v_{0}\right)$. We follow the proof of Theorem 2 by showing that the graphs of the solutions $\tilde{u}^{ \pm}$intersect specified rectangles. For example in the case of $\tilde{u}^{+}$, we want to show that this graph intersects the rectangle $[x, x+a B t] \times[u(x, t), u(x, t)+$ $a(1+t M B)]$. Therefore, we want to prove that for any $y=y(x, t)$ and some $\tilde{y}=\tilde{y}(x+a B t, t)$,

$$
\tilde{u}_{0}(\tilde{y}) \leq u(x, t)+a(1+t B M)=u_{0}(y)+a(1+t B M) .
$$

Since $(x-y) / t=f^{\prime}\left(u_{0}(y)\right)$ and $(x+a B t-\tilde{y}) / t=f^{\prime}\left(\tilde{u}_{0}^{+}(\tilde{y})\right)$, we have either $\tilde{y}-y \leq a t B$, or $f^{\prime}\left(\tilde{u}_{0}^{+}(\tilde{y})\right) \leq f^{\prime}\left(u_{0}(y)\right)$. If the latter holds, the convexity of $f$ implies $\tilde{u}_{0}^{+}(\tilde{y}) \leq u_{0}(y)$ and $(38)$ is done. On the other hand, if we assume that $\tilde{y}-y \leq a t B$, we use one-sided Lipschitz condition to find

$$
\tilde{u}_{0}^{+}(\tilde{y})-u_{0}(y)=a+u_{0}(\tilde{y})-u_{0}(y) \leq a+M(\tilde{y}-y)
$$




$$
\leq a(1+t B M)
$$

which is (38). The case $\tilde{u}_{0}^{-}=u_{0}-a$ is treated similarly and the proof of the Theorem is completed.

Remark 3: the $L^{1}$ distance $\|v-w\|_{L^{1}}$ between two admissible functions $v$ and $w$ is always controlled by the Hausdorff distance $d(v, w)$ provided that one of them (say $v$ ) satisfies a one sided condition (9). Indeed with $\delta=d(v, w)$, we define $v^{+}:=v(x+e \delta)-\tilde{M} \delta$ and $v^{-}:=w(x-e \delta)+\tilde{M} \delta$ as in the proof of Theorem 1 , and since $v^{-} \leq v, w \leq v^{+}$, we have

$$
\|v-w\|_{L^{1}} \leq\left\|v^{+}-v^{-}\right\|_{L^{1}(S)},
$$

where $S=\operatorname{Supp}(v) \cup \operatorname{Supp}(w)$. Using the fact that $v$ has bounded total variation $|v|_{B V} \leq 2 M|S|$, we have

$$
\left\|v^{+}-v^{-}\right\|_{L^{1}(S)} \leq 2 \tilde{M}|S| \delta+\|v(\cdot+e \delta)-v(\cdot-e \delta)\|_{L^{1}} \leq 2(\tilde{M}+M)|S| \delta,
$$

and therefore

$$
\|v-w\|_{L^{1}} \leq 2(\tilde{M}+M)|S| d(v, w) .
$$

\section{Hausdorff stability with respect to a per- turbation of the flux}

We now consider the solution $v$ of a perturbation of (1) according to

$$
\partial_{t} v+\operatorname{Div}_{x}(g(v))=0, \quad t>0, \quad v(x, t=0)=u_{0}(x),
$$

where $g$ is a perturbation of $f$. We assume that both $f$ and $g$ satisfiy

$$
0<A \leq g^{\prime \prime}(z), f^{\prime \prime}(z) \leq B, \quad z \in \mathbb{R} .
$$

We then have the following result.

Theorem 4. Let the fluxes $f, g$ satisfy (43) and let $u_{0}$ be any admissible function. If $u$ and $v$ are the solutions of (1) and (42) respectively with the same initial value $u_{0}$, then, we have the estimate

$$
d(u, v) \leq C(t)\left\|f^{\prime}-g^{\prime}\right\|_{L^{\infty}}, \quad t>0
$$


with $C(t)=\left[(t B / A)^{2}+\Lambda^{2}\right]^{1 / 2}$ and $\Lambda:=(B / A+1) / A$.

Proof : As earlier, we define

$$
\varepsilon:=\left\|f^{\prime}-g^{\prime}\right\|_{L^{\infty}}
$$

Let us first observe that it is enough to prove that

$$
\eta[v(x+\eta t \varepsilon B / A, t)-u(x, t)] \leq \varepsilon \Lambda, \quad \text { for } \quad \eta \in\{-1,1\} .
$$

with $\Lambda=(B / A+1) / A$ as defined above. Indeed, these two estimates inform us that the graph of $v(\cdot, t)$ passes through the rectangle with center $\{x, u(x, t)\}$ and upper right corner $\{x+t \varepsilon B / A, u(x, t)+\varepsilon \Lambda\}$. Hence,

$$
\operatorname{dist}\left(\{x, u(x, t)\}, G_{v}\right) \leq \varepsilon\left[(t B / A)^{2}+\Lambda^{2}\right]^{1 / 2},
$$

and reversing the roles of $u$ and $v$ shows that the same inequality holds for $\operatorname{dist}\left(\{x, v(x, t)\}, G_{u}\right)$.

It remains to prove (46). For this, we let $y=y(x, t)$ and $\tilde{y}_{\eta}=\tilde{y}(x+$ $\eta \varepsilon t B / A, t)$. Since $u(x, t)=u_{0}(y)$ and $v(x+\eta t \varepsilon B / A, t)=u_{0}\left(\tilde{y}_{\eta}\right)$, we need to show that

$$
\eta\left[u_{0}\left(\tilde{y}_{\eta}\right)-u_{0}(y)\right] \leq \epsilon \Lambda .
$$

This inequality is trivial if $\eta\left[u_{0}\left(\tilde{y}_{\eta}\right)-u_{0}(y)\right] \leq 0$. In the remaining case, we write $x-y=t f^{\prime}\left(u_{0}(y)\right)$ and $x+\eta \varepsilon t B / A-\tilde{y}_{\eta}=t g^{\prime}\left(u_{0}\left(\tilde{y}_{\eta}\right)\right)$. From Lemma 1 , part (i), we know that we can choose $\tilde{y}_{\eta}$ such that $\eta\left(\tilde{y}_{\eta}-y\right) \geq 0$ and therefore

$$
\varepsilon t B / A+\eta t\left[f^{\prime}\left(u_{0}(y)\right)-g^{\prime}\left(u_{0}\left(\tilde{y}_{\eta}\right)\right)\right]=\eta\left(\tilde{y}_{\eta}-y\right) \geq 0 .
$$

That is,

$$
\eta\left[g^{\prime}\left(u_{0}\left(\tilde{y}_{\eta}\right)-f^{\prime}\left(u_{0}(y)\right)\right] \leq \varepsilon B / A\right.
$$

Using this with $(27)$ gives

$$
\eta\left[f^{\prime}\left(u_{0}\left(\tilde{y}_{\eta}\right)\right)-f^{\prime}\left(u_{0}(y)\right)\right] \leq \varepsilon(B / A+1)
$$

In the remaining case $\eta\left[u_{0}\left(\tilde{y}_{\eta}\right)-u_{0}(y)\right] \geq 0$, the strict convexity (43) of $f$ gives that the left side of $(51)$ is larger than $\eta\left[u_{0}\left(\tilde{y}_{\eta}\right)-u_{0}(y)\right] / A$ and so the proof is complete. 


\section{Non-convex fluxes}

In this section, we shall prove that Hausdorff stability cannot hold when $f^{\prime \prime}(u)$ has a sign change. The idea for constructing counterexamples to the Hausdorff stability is to take a smooth initial condition $u_{0}$ which gives a solution $u$ which for some later time $T>0$ has two independent shock waves moving towards one another. The topological character of the solution changes when the two shocks collide. Perturbing the initial condition has the effect of changing the collision time and destroys Hausdorff stability. The details are given in the following theorem.

Theorem 5. Assume that $f^{\prime \prime}$ is $C^{1}$ and has a sign change. Then there exists $K, T>0$ and a smooth initial data $u_{0}$ such that for all $\delta>0$, there exists another smooth initial data $v_{0}$ such that $d\left(u_{0}, v_{0}\right) \leq \delta$ and such that at time $T$, the entropy solutions of (1) with such initial data satisfy $d(u, v) \geq K$.

Proof: Without loss of generality, we assume that there exist three values $a<b<c$ such that $f^{\prime \prime}(a)>0, f^{\prime \prime}(b)=0$ and $f^{\prime \prime}(c)<0$, and such that that $f^{\prime \prime} \geq 0$ over $[a, b]$ and $f^{\prime \prime} \leq 0$ over $[b, c]$. We can also assume without loss of generality that $f^{\prime}(a)=0$, since the entropy solutions of (1) with $f^{\prime}$ replaced by $f^{\prime}-f^{\prime}(a)$ are given by $\tilde{u}(x, t):=u\left(x-f^{\prime}(a) t, t\right)$ and $\tilde{v}(x, t):=v\left(x-f^{\prime}(a) t, t\right)$ and that obviously $d(u, v)=d(\tilde{u}, \tilde{v})$.

Consider a smooth initial data $u_{0}^{l}$ such that $u_{0}^{l}(x)=b$ for $x \leq 0, u_{0}^{l}(x)=a$ for $x \geq 1$ and $u_{0}^{l}$ is nonincreasing over $[0,1]$. After some finite time $T_{1}$, this initial data becomes a pure shock wave : for $t>T_{1}$, one has

$$
u^{l}(x, t)=b \chi_{x \leq y^{l}+v^{l} t}(x)+a \chi_{x \geq y^{l}+v^{l} t}(x),
$$

where $y^{l}$ is fixed and $v^{l}=(f(b)-f(a)) /(b-a)>0$.

Consider next a smooth initial data $u_{0}^{r}$ such that $u_{0}^{r}(x)=c$ for $x \geq 0$, $u_{0}^{r}(x)=a$ for $x \leq-1$ and $u_{0}^{r}$ is nondecreasing over $[-1,0]$. By choosing $a$ sufficiently close to $b$, we may assume that $(f(c)-f(a)) /(c-a)<0$. It follows that after some finite time $T_{2}$, this initial data also becomes a pure shock wave : for $t>T_{2}$, one has

$$
u^{r}(x, t)=a \chi_{x \leq y^{r}+v^{r} t}(x)+c \chi_{x \geq y^{r}+v^{r} t}(x),
$$

where $y^{r}$ is fixed and $v^{r}=(f(c)-f(a)) /(c-a)<0$. 
We now define the initial data $u^{0}$ by

$$
u_{0}(x):=u_{0}^{l}(x)+u_{0}^{r}(x-z),
$$

where $z$ is a real number which satisfies $z>y^{l}-y^{r}+\left(v^{l}-v^{r}\right) \max \left(T_{1}, T_{2}\right)$. This condition ensures that the two shock waves begin to interact only after they are completely developed: for $\max \left(T_{1}, T_{2}\right) \leq t \leq T_{3}$ with $y^{r}-y^{l}+\left(v^{r}-v^{l}\right) T_{3}=$ $z$, we have

$$
u(x, t)=u^{l}(x, t)+u^{r}(x-z, t) .
$$

At time $T_{3}$, the two waves meet at the point $y=y^{l}+v^{l} T_{3}=y^{r}+v^{r} T_{3}-z$, and for $t>T_{3}$, the solution becomes a single shock wave

$$
u(x, t)=b \chi_{x \leq y+v\left(t-T_{3}\right)}(x)+c \chi_{x \geq y+v\left(t-T_{3}\right)}(x),
$$

with $v=(f(c)-f(b)) /(c-b)<0$.

For any arbitrarily small $\delta>0$, we define

$$
v_{0}(x):=u_{0}^{l}(x+\delta)+u_{0}^{r}(x-z)
$$

which is a perturbation of $u_{0}$. Then,

$$
v(x, t)=u^{l}(x+\delta, t)+u^{r}(x-z, t),
$$

for $\max \left(T_{1}, T_{2}\right) \leq t \leq T_{3}^{\delta}$ with $y^{r}-y^{l}+\left(v^{r}-v^{l}\right) T_{3}^{\delta}=z-\delta$. It follows clearly that for $T_{3}<t<T_{3}^{\delta}$, we have $d(u, v) \geq K=(b-a)$ while $d\left(u_{0}, v_{0}\right) \leq \delta$, which concludes the proof.

\section{The multidimensional case}

We finally consider the multidimensional case, namely equation (1) with a flux function $f(u)=\left(f_{1}(u), \cdots, f_{d}(u)\right)$. We say that a multivariate function $v$ is one-sided Lipschitz in the direction of $b \in \mathbb{R}^{\mathrm{d}}$ if

$$
v(x+s b)-v(x) \leq M|b| s, \quad s>0
$$

with $M>0$ a fixed constant. The following theorem shows that Hausdorff stability only holds in the case of fluxes which are inherently one dimensional 
up to a linear advection part.

Theorem 6. Assume that the flux $f$ is of the form $f=\left(g_{1}, \cdots, g_{d}\right)+a g$ where the $g_{i}$ are affine functions, a is a fixed unit vector of $\mathbb{R}^{\mathrm{d}}$ and $g$ is a $C^{2}$ function of one variable such that $0 \leq g^{\prime \prime}(u) \leq B$.

If $u_{0}$ is continuous and satisfies the one-sided Lipschitz condition (59) in all directions $b$ such that $\langle b, a\rangle \geq 0$, then for each continuous $v_{0}$ we have

$$
d(u, v) \leq C(t) d\left(u_{0}, v_{0}\right),
$$

for $t>0$, with $C(t)=\left[(1+\tilde{M} B t)^{2}+(\tilde{M}(1+t B M))^{2}\right]^{1 / 2}$ and $\tilde{M}=(1+$ $\left.M^{2}\right)^{1 / 2}+M$.

If the flux $f$ is not of the above form, then there exists $K, T>0$ and a smooth initial data $u_{0}$ with the above properties such that for all $\delta>0$, there exists another smooth initial data $v_{0}$ such that $d\left(u_{0}, v_{0}\right) \leq \delta$ and such that at time $T$, the entropy solutions of (1) with initial data $u_{0}, v_{0}$ satisfy $d(u, v) \geq K$.

Proof : For the first part of theorem, we can assume that $g_{i}=0$ for all $i$, since we have $u(x, t)=\tilde{u}\left(x-\left(g_{1}, \cdots, g_{d}\right) t, t\right)$ and $v(x, t)=\tilde{v}\left(x-\left(g_{1}, \cdots, g_{d}\right) t, t\right)$, with $\tilde{u}$ and $\tilde{v}$ solutions of (1) with $f-\left(g_{1}, \cdots, g_{d}\right)$ in place of $f$ and since clearly $d(u, v)=d(\tilde{u}, \tilde{v})$. Setting $u_{0}^{ \pm}:=u_{0}(\cdot \mp a \delta) \pm \tilde{M} \delta$, we observe that condition (59) in all directions $b$ such that $\langle b, a\rangle \geq 0$ implies that if $\delta:=d\left(u_{0}, v_{0}\right)$ then

$$
u_{0}^{-} \leq u_{0}, v_{0} \leq u_{0}^{+} .
$$

We denote by $u^{+}$and $u^{-}$the solutions corresponding to these initial data.

We now remark that for all $x \in(a \mathbb{R})^{\perp}$, the function $u^{(x)}(s, t):=u(x+$ $s a, t)$ with $s \in \mathbb{R}$ is an entropy solution of the one dimensional conservation law (1) with $g$ as the flux function. From the one dimensional theory, we know that $s \mapsto u^{(x)}(s, t)$ is admissible in the sense given in the introduction and we can define its graph $G_{u^{(x)}}$. Moreover, since $u_{0}$ is assumed to be continuous, we have

$$
d\left(u^{(x)}(\cdot, t), u^{(\tilde{x})}(\cdot, t)\right) \leq C(t) d\left(u^{(x)}(\cdot, 0), u^{(\tilde{x})}(\cdot, t)\right) \rightarrow 0 \text { as } \tilde{x} \rightarrow x .
$$

This shows that at any time $t>0$, the graph of $G_{u}$ of $u$ can be defined from the graphs $G_{u^{(x)}}$ by

$$
G_{u}=\cup\left\{(x+a s, y) ;(s, y) \in G_{u^{(x)}} ; x \in(a \mathbb{R})^{\perp},\right.
$$


i.e. $G_{u}$ is the union of all its one dimensional sections in the direction $a$. The graphs of $v, u^{+}$and $u^{-}$can be defined in a similar way. We can then use Theorem 3 to derive

$$
\begin{aligned}
d(u, v) & \leq \max \left\{d\left(u^{-}, u\right), d\left(u^{+}, u\right)\right\} \\
& \leq \sup _{x \in(a \mathbb{R})^{\perp}} \max \left\{d\left(\left(u^{(x)}\right)^{-}, u^{(x)}\right), d\left(\left(u^{(x)}\right)^{+}, u^{(x)}\right)\right\} \\
& \leq C(t) \delta=C(t) d\left(u_{0}, v_{0}\right),
\end{aligned}
$$

which concludes the first part of the theorem.

To see that the condition on $f$ is necessary, we suppose that $f$ is any flux function. If $b$ is any vector of unit length, we consider the function

$$
u(x, t)=u^{(b)}(\langle x, b\rangle, t),
$$

with $u^{(b)}$ a one-dimensional entropy solution of (1) with $f_{b}:=\langle f, b\rangle$ as a flux function and some $u_{0}^{(b)}$ as the initial value. Then, one easily verifies that $u$ is a solution to (1) with flux $f$ and initial condition $u_{0}(x)=u_{0}^{(b)}(\langle b, x\rangle)$. At any time $t>0$, the function $u^{(b)}$ is admissible in the sense given in the introduction, and this allows us to define its graph as well as the graph of $u$. According to Theorem 5, we can construct unstable solutions in the Hausdorff metric as soon as $f_{b}^{\prime \prime}$ has a sign change. This means that stability in the Hausdorff metric requires that for all unit vectors $b$, the function $\left\langle f^{\prime \prime}, b\right\rangle=\sum b_{i} f_{i}^{\prime \prime}$ has no sign change. This implies that $f^{\prime \prime}$ is of the form $a g^{\prime \prime}$ with $a$ a fixed unit vector and $g^{\prime \prime}$ a positive one-dimensional function, and therefore $f$ has the announced form.

\section{References}

[1] M. Campos-Pinto, A. Cohen and P. Petrushev, High order geometric smoothness for conservation laws, preprint Laboratoire J.-L. Lions, Université Pierre et Marie Curie, Paris, 2003.

[2] C. Dafermos, Hyperbolic conservation laws in continuum physcs, Grundlehren der mathematischen Wissenschaften 325, Springer Verlag, Berlin, 2000. 
[3] R. DeVore and B. Lucier, High order regularity for conservation laws, Indiana Math. J. 39, No. 2, 1990, 413-430.

[4] E. Godlewski and P.-A. Raviart, Numerical approximation of hyperbolic systems of conservation laws, Applied Mathematical Sciences, 118. Springer-Verlag, New York, 1996.

[5] S.N. Kruzkov, First order quasilinear equations in several independent variables, Math. USSR Sb. 10, 1970, 217-243.

[6] N.N. Kuznetsov, Accuracy of some approximate methods for computing the weak solutions of a first-order quasi-linear equation, USSR Comput. Math. and Math. Phys. 16, 1976, 105-119.

[7] P.D. Lax, Hyperbolic Systems of Conservation Laws and the Mathematical Theory of Shock Waves, Vol. 11, Regional Conference Series in Applied Mathematics, SIAM, Philadelphia, 1973.

[8] R. LeVeque, Numerical methods for conservation laws, Lectures in Mathematics ETH Zurich. Birkhauser Verlag, Basel, 1990.

[9] B. Lucier, A moving mesh numerical method for hyperbolic conservation laws, Math. Comp. 46, 1986, No. 173, 59-69.

[10] E. Tadmor, Local error estimates for discontinuous solutions of nonlinear hyperbolic equations, SIAM J. Numer. Anal. 28, 1991, 891-906.

[11] E. Tadmor and T. Tang, Pointwise convergence rate for scalar conservation laws with piecewise smooth solutions, SIAM J. Numer. Anal. 36, 1999, 1739-1758.

[12] E. Tadmor and T. Tang, Pointwise error estimates for relaxation approximations to conservation laws, SIAM J. Math. Anal. 32, 2001,870-886. 